\title{
Effect of Moisture Regimes, FYM and Levels of P Carriers on Yield, Quality and P Uptake by Wheat in Loamy Sand
}

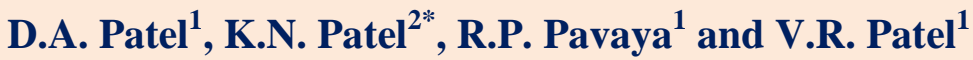 \\ ${ }^{1}$ C. P. College of Agriculture, S.D.A.U., Sardarkrushinagar, Gujarat, India \\ ${ }^{2}$ Centre for Research on Integrated Farming Systems, S.D.A.U., \\ Sardarkrushinagar, Gujarat, India \\ *Corresponding author
}

\section{A B S T R A C T}

In field study, total 36 treatment combinations consisting of three moisture regimes as main plot treatment and 12 combinations of two levels of FYM, three levels of P carriers as sub-plot treatments evaluated in split plot design with three replications using wheat variety GW-451 as test crop. The results revealed that

Keywords

Moisture regimes,

$\mathrm{P}$ carrier and

levels of $\mathrm{P}$

\section{Article Info}

Accepted:

10 July 2019

Available Online:

10 August 2019 the maintenance of moisture between 100 and $75 \%$ available water content $\left(\mathrm{W}_{1}\right)$ significantly increased the length of earhead, number of grains/earhead, 1000-grain weight, grain and straw yield as well as gluten content. The treatments $\mathrm{W}_{1}$ and $\mathrm{W}_{2}$ resulted in 22.11 and 18.45 per cent higher grain yield as well as 23.56 and 19.91 per cent higher straw yield, respectively over $\mathrm{W}_{3}$ treatment. Whereas, maintenance of soil moisture between 100 and $25 \% \mathrm{AWC}\left(\mathrm{W}_{3}\right)$ resulted significantly higher $\mathrm{P}$ content in grain and straw of wheat. Application of phosphorus @ $90 \mathrm{~kg} \mathrm{P}_{2} \mathrm{O}_{5}$ as MAP with $10 \mathrm{t} / \mathrm{ha} \mathrm{FYM}\left(\mathrm{P}_{3} \mathrm{~S}_{1} \mathrm{M}_{1}\right)$ gave significantly higher length of earhead, number of grains/earhead, 1000-grain weight, grain yield and application of 60 $\mathrm{kg} \mathrm{P}_{2} \mathrm{O}_{5} / \mathrm{ha}$ as DAP with $10 \mathrm{t} \mathrm{FYM/ha} \mathrm{registering} 4660 \mathrm{~kg} / \mathrm{ha}$ grain yield which was at par with $\mathrm{P}_{3} \mathrm{~S}_{1} \mathrm{M}_{1}$ while, Treatment combinations $\mathrm{P}_{3} \mathrm{~S}_{2} \mathrm{M}_{1}$ showed significantly higher $\mathrm{P}$ content in grain and straw, but it was at par with $\mathrm{P}_{3} \mathrm{~S}_{1} \mathrm{M}_{1}$ and $\mathrm{P}_{2} \mathrm{~S}_{2} \mathrm{M}_{1}$. While significantly the highest $\mathrm{P}$ uptake by grain, straw and total $\mathrm{P}$ uptake by wheat was recorded by $\mathrm{P}_{3} \mathrm{~S}_{1} \mathrm{M}_{1}$, but it was at par with $\mathrm{P}_{2} \mathrm{~S}_{2} \mathrm{M}_{1}$. The interaction $\mathrm{W}_{2} \times \mathrm{P}_{3} \mathrm{~S}_{1} \mathrm{M}_{1}$ recorded maximum length of earhead $(11.09 \mathrm{~cm})$, number of grains/earhead (68), 1000-grain weight $(73.18 \mathrm{~g})$, grain $(5210 \mathrm{~kg} / \mathrm{ha})$ and straw $(11969 \mathrm{~kg} / \mathrm{ha})$ yield of wheat and gluten content $(39.42 \%)$ in grain. The lowest value for bulk density $\left(1.61 \mathrm{Mg} / \mathrm{m}^{3}\right)$ and the highest value for maximum water holding capacity $(22.49 \%)$ of soil after harvest were recorded by $\mathrm{P}_{2} \mathrm{~S}_{1} \mathrm{M}_{1}$. Treatment combination $\mathrm{P}_{1} \mathrm{~S}_{2} \mathrm{M}_{1}$ recorded significantly higher available $\mathrm{N}(177.30 \mathrm{~kg} / \mathrm{ha}), \mathrm{P}_{2} \mathrm{O}_{5}(40.7 \mathrm{~kg} / \mathrm{ha})$ and $\mathrm{K}_{2} \mathrm{O}(207.57 \mathrm{~kg} / \mathrm{ha})$ in soil after harvest.

\section{Introduction}

An ideal moisture regime favors the absorption of mobile nutrients. The accessibility of immobile nutrient like $\mathrm{PO}_{4}{ }^{-3}$ is increased due to more root development in a soil having low suction of moisture. A favorable moisture regime also, increases the uptake of available nutrients. An ideal moisture regime in combination with optimum dose of nutrient not only increases the yield, but also the nutrient use efficiency. The extent of response to phosphorus fertilizer application in relation to moisture is determined by the rate and distance of diffusion of phosphate ion to come in contact with root surface release of non-labile phosphorus and mineralization of organic-P. Phosphate ion is partially immobile and hence, it's placement in root zone/moist layer is must for its efficient utilization. In most soils, phosphorus availability is the highest in the 
surface plow layer and much lower in the subsoil. Therefore, during a period of dry weather, if the surface soil becomes dry, plant suffers from a phosphorus deficiency even though moisture is still available in the subsoil and plants show no water stress.

This is a potential threat to sustainable crop production. Almost 80 per cent soils in India are either low or medium in $\mathrm{P}$ fertility status, which reinforces the need for $\mathrm{P}$ application in the form of organic manures, green manures and phosphatic fertilizers to maximize the crop yields (Sanyal et al., 2015). In Gujarat, 60 per cent soils are classified as deficient in available $\mathrm{P}$ status.

The GAU has made 38 crop specific $\mathrm{P}$ recommendations. Instances of erratic response to $\mathrm{P}$ application by different crops or crop sequences are quite common. The state average of $\mathrm{P}$ consumption is only $18.1 \mathrm{~kg}$ $\mathrm{P}_{2} \mathrm{O}_{5} /$ ha (Patel et al., 1994).

Wheat [Triticum aestivum (L.)] has been described as "king of cereals" and one of the most important staple food crop cultivated in 43 countries of the world. Wheat has its own outstanding importance as a human food; the nutritive value of wheat is fairly high as compared to other cereals. It contains 11.80, $1.50,71.20,1.50,0.50,0.32$, per cent, fat, carbohydrate, mineral matter, calcium and phosphorus, respectively (Swaminathan et al., 1981).

Gujarat occupies in area of 1.05 million hectares with a production of 3.13 million tones and productivity of $2986 \mathrm{~kg} / \mathrm{ha}$. The most important wheat growing districts of the state are Junagadh, Ahmedabad, Sabarkantha, Rajkot, Kheda, Banaskantha, Mehsana and Bhavnagar.

The combined use of moisture regimes, FYM and levels of $\mathrm{P}$ carriers, the immobile properties of $\mathrm{P}$ can be exploited by mobilizing native plant unavailable $\mathrm{P}$ to available form so that, it may be recycled the native $\mathrm{P}$ and minimize $\mathrm{P}$ fertilizer particularly in wheat crop.

\section{Materials and Methods}

\section{Climate and weather conditions}

The maximum and minimum temperature ranged between 24.5 to $36.40^{\circ} \mathrm{C}$ and 6.8 to $18.7^{\circ} \mathrm{C}$, respectively no rainfall was received. The mean relative humidity recorded at 7.40 am ranged between 22.4 to 89.3 per cent and the mean sunshine hours ranged between 7.7 to 9.4 hours during the crop growing period.

The overall climatological data indicated that the weather conditions were observed normal and favorable for the satisfactory growth and development of the crop during the rabi, 2017-18.

\section{Physico-chemical properties of soil}

The representative soil sample was analyzed for different physico-chemical characteristics (Table 1).

\section{Irrigation management}

Two common irrigations were applied, first after sowing for better germination and second at 21 DAS i.e. crown root initiation stage of wheat crop.

After that according to treatments (moisture regimes), the irrigations were given when the moisture content in the soil was reached to 7.66, 6.60 and 5.54 per cent, which represent the availability of soil moisture at 75, 50 and 25 per cent, respectively. Periodically, the soil samples for moisture were taken up to a depth of $30 \mathrm{~cm}$ for determining the needs of irrigation. 


\section{Collection and preparation of plant and soil samples for chemical analysis}

\section{Plant samples}

Representative plant samples were collected at the time of harvesting of crop. Grain and straw yield was recorded. The plant samples were washed with tap water followed by distilled water and dried in oven at $70^{\circ} \mathrm{C}$ till they attained constant weight.

The oven dried plant samples were ground in grinding mill. Finally, the powdered samples were stored in labeled polythene bags for chemical analysis than Vanadomolybdo phosphoric acid yellow colour method was used for determination of P (Jackson, 1978).

\section{Soil samples}

To assess the nutrient status of soil after harvest, a representative soil samples (0-15 $\mathrm{cm})$ from three spots of each net plot after harvest of wheat crop were collected, composited and air dried in shade (Experimental details are given in table 4).

These samples were then ground using wooden mortar and pestle and passed through $2 \mathrm{~mm}$ sieve and were analysed for organic carbon, available $\mathrm{N}, \mathrm{P}_{2} \mathrm{O}_{5}, \mathrm{~K}_{2} \mathrm{O}, \mathrm{EC}$, pH, bulk density and maximum water holding capacity as per standard analytical methods listed in Table 3.

\section{Nutrient content and uptake}

The concentration of the phosphorus was determined in grain and straw.

The uptake of $\mathrm{P}$ was computed by using the following formula.

Phosphorus content $(\%) \times$ Yield $(\mathrm{kg} / \mathrm{ha})$ Phosphorus uptake $(\mathrm{kg} / \mathrm{ha})=$

\section{Results and Discussion}

\section{Yield attributes and yield}

Among the levels of $\mathrm{P}$ carriers with and without application of FYM, combination of $\mathrm{P}_{3} \mathrm{~S}_{1} \mathrm{M}_{1}$ showed significantly higher length of earhead (10.02 cm), grain/earhead (61) and 1000 -grain weight $(65.68 \mathrm{~g})$ as compared to other treatment combinations except $\mathrm{P}_{2} \mathrm{~S}_{2} \mathrm{M}_{1}$ which was at par with $\mathrm{P}_{3} \mathrm{~S}_{1} \mathrm{M}_{1}$ (Table 6). As far as interaction effect between $\mathrm{W} \times \mathrm{PSM}$ is concerned, $\mathrm{W}_{2} \mathrm{P}_{3} \mathrm{~S}_{1} \mathrm{M}_{1}$ ranked at top bearing the highest length of earhead $(11.09 \mathrm{~cm})$, grain/earhead (68) and 1000-grain weight $(73.18 \mathrm{~g})$. As far as the effect of different combinations of FYM and levels of P carriers on wheat grain and straw yield was concerned (Table 6), it was observed that yield of grain with FYM and application of P @ $90 \mathrm{~kg} / \mathrm{ha}$ in the form of MAP $\left(\mathrm{P}_{3} \mathrm{~S}_{1} \mathrm{M}_{1}\right)$ was significantly higher $(4708 \mathrm{~kg} / \mathrm{ha})$ over all the treatment combinations except $\mathrm{P}_{2} \mathrm{~S}_{2} \mathrm{M}_{1}(4660 \mathrm{~kg} / \mathrm{ha})$. The treatment combination $\mathrm{P}_{3} \mathrm{~S}_{1} \mathrm{M}_{1}$ and $\mathrm{P}_{2} \mathrm{~S}_{2} \mathrm{M}_{1}$ registered 47.63 and 46.13 per cent higher grain yield of wheat as compared to $\mathrm{P}_{1} \mathrm{~S}_{2} \mathrm{M}_{0}$. The treatment combination $\mathrm{W}_{2} \mathrm{P}_{3} \mathrm{~S}_{1} \mathrm{M}_{1}$ showed the highest yield (5210 $\mathrm{kg} / \mathrm{ha}$ ). The treatment combination $\mathrm{W}_{2} \mathrm{P}_{3} \mathrm{~S}_{1} \mathrm{M}_{1}$ yielded 85. 28 per cent higher grain yield of wheat than $\mathrm{W}_{2} \mathrm{P}_{1} \mathrm{~S}_{2} \mathrm{M}_{0}$. $\mathrm{W}_{1}$ and $\mathrm{W}_{2}$ treatments gave 23.56 and 19.91 per cent more straw yield of wheat as compared to $\mathrm{W}_{3}$. Maximum straw yield $(9383 \mathrm{~kg} / \mathrm{ha})$ was registered by $\mathrm{W}_{1}$ treatment. Application of P @ $60 \mathrm{~kg} / \mathrm{ha}$ in the form of DAP with FYM $\left(\mathrm{P}_{2} \mathrm{~S}_{2} \mathrm{M}_{1}\right)$ was significantly higher straw yield $(10682 \mathrm{~kg} / \mathrm{ha})$ over all the treatment combinations except $\mathrm{P}_{3} \mathrm{~S}_{1} \mathrm{M}_{1}(10532 \mathrm{~kg} / \mathrm{ha})$. The treatments $\mathrm{P}_{2} \mathrm{~S}_{2} \mathrm{M}_{1}$ and $\mathrm{P}_{3} \mathrm{~S}_{1} \mathrm{M}_{1}$ increased the straw yield of wheat to the tune of 45.83 and 43.78 per cent, respectively over $\mathrm{P}_{1} \mathrm{~S}_{2} \mathrm{M}_{0}$. The similar results were also observed by Singh and Agarwal (2005) with the application of FYM @ 10 and $20 \mathrm{t} / \mathrm{ha}$ and observed an increase in grain and straw yield of wheat. Shahi et al., (2015) also 
observed the role of $\mathrm{P}$ levels and FYM on wheat crop. Application of $\mathrm{P}$ levels significantly augmented crop growth and yield of wheat. This might be due to moisture availability for a longer period to the crop along with positive effect of moisture and FYM on P availability in soil. As far as effect of $\mathrm{P}$ levels and sources on wheat yield is concerned, the findings of Al Harbi et al., (2013) also revealed in their studies that an application of $\mathrm{P}$ at a higher level in the form of DAP showed higher and significant response.

\section{Quality parameter}

The interaction effect of treatment combination $\mathrm{W}_{3} \mathrm{P}_{2} \mathrm{~S}_{1} \mathrm{M}_{0}$ showed significantly lowest $(20.47 \%)$ content of gluten in wheat grain (Table 5). Although, the treatment combination $\mathrm{W}_{2} \mathrm{P}_{3} \mathrm{~S}_{1} \mathrm{M}_{1}$ showed the higher gluten content (39.42\%), but it was at par with the combinations $\quad \mathrm{W}_{1} \mathrm{P}_{2} \mathrm{~S}_{2} \mathrm{M}_{1} \quad(35.17 \%)$ $\mathrm{W}_{2} \mathrm{P}_{2} \mathrm{~S}_{2} \mathrm{M}_{1}$ (37.54\%), $\mathrm{W}_{1} \mathrm{P}_{3} \mathrm{~S}_{1} \mathrm{M}_{1}$ (34.99\%), $\mathrm{W}_{2} \mathrm{P}_{2} \mathrm{~S}_{2} \mathrm{M}_{1}(34.89 \%), \mathrm{W}_{2} \mathrm{P}_{1} \mathrm{~S}_{1} \mathrm{M}_{0}(33.87 \%)$ and $\mathrm{W}_{1} \mathrm{P}_{3} \mathrm{~S}_{1} \mathrm{M}_{0}(33.43 \%)$.

In general the combinations involving $\mathrm{M}_{1}$ with $\mathrm{P}$ application at a higher level of moisture, phosphorus availability had a tendency of showing higher gluten content in wheat grains. Delay in irrigation from $70 \mathrm{~mm}$ to $90 \mathrm{~mm}$ evaporation, significantly increased wet gluten by Jazy et al., (2012).

The data exhibited in Table 7 indicates that maintenance of soil moisture between 100-25 per cent, $\left(\mathrm{W}_{3}\right)$ proved its superiority over $\mathrm{W}_{2}$ and $\mathrm{W}_{1}$ as far as, $\mathrm{P}$ content in grain and straw was concerned as, it follows the Jenny's dilution effect phenomena and contained significantly higher $\mathrm{P}$ content in grain $(0.42$ $\%)$ and straw $(0.31 \%)$. Treatment combination $\mathrm{P}_{3} \mathrm{~S}_{2} \mathrm{M}_{1}$ showed significantly higher $\mathrm{P}$ content in wheat grain $(0.52 \%)$ and straw $(0.38 \%)$. Although, it was at par with treatment combinations $\mathrm{P}_{3} \mathrm{~S}_{1} \mathrm{M}_{1}$ and $\mathrm{P}_{2} \mathrm{~S}_{2} \mathrm{M}_{1}$. The perusal of data given in Table 7 suggests that phosphorus uptake by grain and straw did not differ significantly with moisture regimes. The trend of $\mathrm{P}$ uptake by grain and straw is in the order $\mathrm{W}_{1}>\mathrm{W}_{2}>\mathrm{W}_{3}$. Different treatment combinations were found to affect the $P$ uptake by wheat grain and straw significantly. $\mathrm{P}_{3} \mathrm{~S}_{1} \mathrm{M}_{1}$ registered higher $\mathrm{P}$ uptake $(24.10 \mathrm{~kg}$ $\mathrm{P} / \mathrm{ha}$ ) by grain and straw $(39.18 \mathrm{~kg} / \mathrm{ha})$, but it was at par with $\mathrm{P}_{2} \mathrm{~S}_{2} \mathrm{M}_{1}(22.60 \mathrm{~kg} / \mathrm{ha})$ in grain and $(37.75 \mathrm{~kg} / \mathrm{ha})$. It seems that "dilution effect" has played a crucial role in governing the nutrient concentration in the plant parts.

Of course, content as such is deceptive yardstick for nutrient availability as it increase with decrease in the quantity of dry matter produced by a particular crop. Golakiya (1988) reported that $\mathrm{P}$ uptake was decreased by decreasing soil moisture level.

\section{Nutrient status of soil after harvest}

The bulk density and MWHC of soil, which is one of the important criteria of physical fertility of soil, was directly positively related with the amount of organic matter content in the soil (Table 8). Under this experimentation, this phenomenon is also true. The lowest value of BD $\left(1.61 \mathrm{Mg} / \mathrm{m}^{3}\right)$ and highest MWHC $(22.49 \%)$ was obtained under the treatment combination $\mathrm{P}_{2} \mathrm{~S}_{1} \mathrm{M}_{1}$. Although, it was at par with $\mathrm{P}_{1} \mathrm{~S}_{1} \mathrm{M}_{1}, \mathrm{P}_{2} \mathrm{~S}_{2} \mathrm{M}_{1}, \mathrm{P}_{3} \mathrm{~S}_{1} \mathrm{M}_{1}$ and $\mathrm{P}_{3} \mathrm{~S}_{2} \mathrm{M}_{1}$. Numerically higher value under treatment combination $\quad \mathrm{P}_{1} \mathrm{~S}_{1} \mathrm{M}_{1} \quad(0.18 \mathrm{dS} / \mathrm{m}) \quad$ was observed for electrical conductivity (EC) of soil. Treatment combinations $\mathrm{P}_{3} \mathrm{~S}_{1} \mathrm{M}_{1}$ and $\mathrm{P}_{3} \mathrm{~S}_{2} \mathrm{M}_{1}$ registered significantly the lowest soil $\mathrm{pH}$ (7.24) after the harvest of crop, but it was at par with $\mathrm{P}_{2} \mathrm{~S}_{2} \mathrm{M}_{0}$ (7.30), $\mathrm{P}_{2} \mathrm{~S}_{1} \mathrm{M}_{1}$ (7.30), $\mathrm{P}_{1} \mathrm{~S}_{2} \mathrm{M}_{1}$ (7.30) and $\mathrm{P}_{1} \mathrm{~S}_{1} \mathrm{M}_{1}$ (7.33). With respect to organic carbon content in soil after one season of experimentation treatment combination $\mathrm{P}_{3} \mathrm{~S}_{1} \mathrm{M}_{1}$ registered significantly higher organic carbon content $(0.37 \%)$ over other combinations (Table 8). 
Table.1 Initial physico-chemical properties of the surface soil $(0-15 \mathrm{~cm})$ of the experimental plot before sowing of wheat crop

\begin{tabular}{|c|c|}
\hline Properties & Values \\
\hline \multicolumn{2}{|l|}{ A. Mechanical composition } \\
\hline Coarse sand $(\%)$ & 44.76 \\
\hline Fine sand $(\%)$ & 40.18 \\
\hline Silt $(\%)$ & 7.90 \\
\hline Clay $(\%)$ & 7.16 \\
\hline Textural class & Loamy sand \\
\hline Taxonomy & Typics Ustipsamments \\
\hline Depth $(\mathbf{c m})$ & 30 \\
\hline \multicolumn{2}{|l|}{ B. Physical properties } \\
\hline Bulk density $\left(\mathrm{Mg} \mathrm{m}^{-3}\right)$ & 1.65 \\
\hline Infiltration rate $(\mathrm{cm} / \mathrm{hr})$ & 14.8 \\
\hline F.C. $(\%)$ & 8.72 \\
\hline P.W.P. (\%) & 4.48 \\
\hline A.W.C. $(\%)$ & 4.24 \\
\hline M.W.H.C. $(\%)$ & 22.12 \\
\hline \multicolumn{2}{|l|}{ C. Chemical properties } \\
\hline pH $(1: 2: 5)$ at $25^{\circ} \mathrm{C}$ & 7.5 \\
\hline $\mathrm{EC}(1: 2: 5) \mathrm{dSm}^{-1}$ at $25^{\circ} \mathrm{C}$ & 0.18 \\
\hline Exchangeable Ca (meq/100 g) & 5.6 \\
\hline Exchangeable $\mathrm{Mg}$ (meq/100 g) & 0.8 \\
\hline CEC (meq/100 g) & 7.48 \\
\hline Organic carbon $(\%)$ & 0.349 \\
\hline Available N (kg/ha) & 148.0 \\
\hline Available $\mathrm{P}_{2} \mathrm{O}_{5}(\mathrm{~kg} / \mathrm{ha})$ & 33.15 \\
\hline Available $\mathrm{K}_{2} \mathrm{O}$ (kg/ha) & 177.26 \\
\hline
\end{tabular}

Table. 2 Total treatment combinations

\begin{tabular}{|c|c|c|c|}
\hline Treatments & Combinations & Treatments & Combinations \\
\hline $\mathbf{T}_{1}$ & $\mathrm{~W}_{1} \mathrm{P}_{1} \mathrm{~S}_{1} \mathrm{M}_{0}$ & $\mathrm{~T}_{19}$ & $\mathbf{W}_{2} \mathbf{P}_{2} \mathbf{S}_{2} \mathbf{M}_{0}$ \\
\hline $\mathbf{T}_{2}$ & $\mathrm{~W}_{1} \mathrm{P}_{1} \mathrm{~S}_{1} \mathrm{M}_{1}$ & $\mathrm{~T}_{20}$ & $\mathrm{~W}_{2} \mathbf{P}_{2} \mathrm{~S}_{2} \mathrm{M}_{1}$ \\
\hline $\mathbf{T}_{3}$ & $\mathrm{~W}_{1} \mathrm{P}_{1} \mathrm{~S}_{2} \mathrm{M}_{0}$ & $\mathrm{~T}_{21}$ & $\mathbf{W}_{2} \mathbf{P}_{3} \mathbf{S}_{1} \mathbf{M}_{0}$ \\
\hline $\mathbf{T}_{4}$ & $\mathrm{~W}_{1} \mathrm{P}_{1} \mathrm{~S}_{2} \mathrm{M}_{1}$ & $\mathrm{~T}_{22}$ & $\mathbf{W}_{2} \mathbf{P}_{3} \mathbf{S}_{1} \mathbf{M}_{1}$ \\
\hline $\mathbf{T}_{5}$ & $\mathrm{~W}_{1} \mathrm{P}_{2} \mathrm{~S}_{1} \mathrm{M}_{0}$ & $\mathrm{~T}_{23}$ & $\mathbf{W}_{2} \mathbf{P}_{3} \mathbf{S}_{2} \mathbf{M}_{0}$ \\
\hline$T_{6}$ & $\mathrm{~W}_{1} \mathrm{P}_{2} \mathrm{~S}_{1} \mathrm{M}_{1}$ & $\mathrm{~T}_{24}$ & $\mathbf{W}_{2} \mathbf{P}_{3} \mathbf{S}_{2} \mathbf{M}_{1}$ \\
\hline $\mathbf{T}_{7}$ & $\mathrm{~W}_{1} \mathrm{P}_{2} \mathrm{~S}_{2} \mathrm{M}_{0}$ & $\mathrm{~T}_{25}$ & $\mathbf{W}_{3} \mathbf{P}_{1} \mathbf{S}_{1} \mathbf{M}_{0}$ \\
\hline $\mathbf{T}_{8}$ & $\mathrm{~W}_{1} \mathrm{P}_{2} \mathrm{~S}_{2} \mathrm{M}_{1}$ & $\mathrm{~T}_{26}$ & $\mathbf{W}_{3} \mathbf{P}_{1} \mathbf{S}_{1} \mathbf{M}_{1}$ \\
\hline $\mathbf{T}_{9}$ & $\mathrm{~W}_{1} \mathrm{P}_{3} \mathrm{~S}_{1} \mathrm{M}_{0}$ & $\mathrm{~T}_{27}$ & $\mathbf{W}_{3} \mathbf{P}_{1} \mathbf{S}_{2} \mathbf{M}_{0}$ \\
\hline$T_{10}$ & $\mathrm{~W}_{1} \mathrm{P}_{3} \mathrm{~S}_{1} \mathrm{M}_{1}$ & $\mathrm{~T}_{28}$ & $\mathbf{W}_{3} \mathbf{P}_{1} \mathbf{S}_{2} \mathbf{M}_{1}$ \\
\hline $\mathbf{T}_{11}$ & $\mathrm{~W}_{1} \mathrm{P}_{3} \mathrm{~S}_{2} \mathrm{M}_{0}$ & $\mathrm{~T}_{29}$ & $\mathbf{W}_{3} \mathbf{P}_{2} \mathbf{S}_{1} \mathbf{M}_{0}$ \\
\hline $\mathbf{T}_{12}$ & $\mathrm{~W}_{1} \mathrm{P}_{3} \mathrm{~S}_{2} \mathrm{M}_{1}$ & $\mathrm{~T}_{30}$ & $\mathbf{W}_{3} \mathbf{P}_{2} \mathbf{S}_{1} \mathbf{M}_{1}$ \\
\hline $\mathbf{T}_{13}$ & $\mathrm{~W}_{2} \mathrm{P}_{1} \mathrm{~S}_{1} \mathrm{M}_{0}$ & $\mathrm{~T}_{31}$ & $\mathrm{~W}_{3} \mathbf{P}_{2} \mathbf{S}_{2} \mathbf{M}_{0}$ \\
\hline$T_{14}$ & $\mathrm{~W}_{2} \mathrm{P}_{1} \mathrm{~S}_{1} \mathrm{M}_{1}$ & $\mathrm{~T}_{32}$ & $\mathbf{W}_{3} \mathbf{P}_{2} \mathbf{S}_{2} \mathbf{M}_{1}$ \\
\hline$T_{15}$ & $\mathrm{~W}_{2} \mathrm{P}_{1} \mathrm{~S}_{2} \mathrm{M}_{0}$ & $\mathrm{~T}_{33}$ & $\mathbf{W}_{3} \mathbf{P}_{3} \mathbf{S}_{1} \mathbf{M}_{0}$ \\
\hline$T_{16}$ & $\mathrm{~W}_{2} \mathrm{P}_{1} \mathrm{~S}_{2} \mathrm{M}_{1}$ & $\mathrm{~T}_{34}$ & $\mathbf{W}_{3} \mathbf{P}_{3} \mathbf{S}_{1} \mathbf{M}_{1}$ \\
\hline $\mathbf{T}_{17}$ & $\mathrm{~W}_{2} \mathrm{P}_{2} \mathrm{~S}_{1} \mathrm{M}_{0}$ & $\mathrm{~T}_{35}$ & $\mathbf{W}_{3} \mathbf{P}_{3} \mathbf{S}_{2} \mathbf{M}_{0}$ \\
\hline $\mathbf{T}_{18}$ & $\mathrm{~W}_{2} \mathbf{P}_{2} \mathrm{~S}_{1} \mathrm{M}_{1}$ & $\mathbf{T}_{36}$ & $\mathbf{W}_{3} \mathbf{P}_{3} S_{2} M_{1}$ \\
\hline
\end{tabular}




\begin{tabular}{|l|l|l|l|}
\hline \multicolumn{5}{|c|}{ Table.3 Methods followed for soil analysis } \\
\hline Sr. No. & Element & Methods & Reference(s) \\
\hline $\mathbf{1}$ & Organic carbon & Walkley and Black titration & Jackson (1978). \\
\hline $\mathbf{2}$ & Available $\mathrm{N}$ & Alkaline $\mathrm{KMnO}_{4}$ method & $\begin{array}{l}\text { (Subbiah and Asija, } \\
\text { 1956). }\end{array}$ \\
\hline $\mathbf{3}$ & Available $\mathrm{P}_{2} \mathrm{O}_{5}$ & Extraction: $0.5 \mathrm{M} \mathrm{NaHCO}_{3}(\mathrm{pH} 7.0)$ & Olsen's et al., (1954). \\
\hline $\mathbf{4}$ & Available $\mathrm{K}_{2} \mathrm{O}$ & Flame photometric method $\mathrm{NH}_{4} \mathrm{OAC}(\mathrm{pH} \mathrm{7.0)}$ & Jackson (1973). \\
\hline $\mathbf{5}$ & Soil reaction $(\mathrm{pH})$ & $1: 2.5$ on Beckmen $\mathrm{pH}$ meter & (Jackson, 1973). \\
\hline $\mathbf{6}$ & Electrical Conductivity (EC) & $1: 2.5$ with water & (Jackson, 1973). \\
\hline $\mathbf{7}$ & Bulk Density (BD) & Core method & (Culley, 1993). \\
\hline $\mathbf{8}$ & $\begin{array}{l}\text { Water Holding Capacity } \\
\text { (WHC) }\end{array}$ & Gravimetric method & (Piper, 1966). \\
\hline
\end{tabular}

Table.4 Experimental details

\begin{tabular}{|c|c|c|c|}
\hline \multirow[t]{2}{*}{ A } & \multicolumn{3}{|c|}{ Main plot treatment : 03} \\
\hline & $\mathrm{W}_{1}$ & $=$ & Maintenance of moisture between 100 and $75 \%$ availability at $0-30 \mathrm{~cm}$ depth \\
\hline & $\mathbf{W}_{2}$ & $=$ & Maintenance of moisture between 100 and $50 \%$ availability at $0-30 \mathrm{~cm}$ depth \\
\hline & $\mathbf{W}_{3}$ & $=$ & Maintenance of moisture between 100 and $25 \%$ availability at $0-30 \mathrm{~cm}$ depth \\
\hline \multirow[t]{11}{*}{ B } & \multicolumn{3}{|c|}{ Sub-plot treatments : } \\
\hline & \multicolumn{3}{|c|}{ a. Levels of $P: 03$} \\
\hline & $\mathbf{P}_{1}$ & $=$ & $30 \mathrm{~kg} \mathrm{P}_{2} \mathrm{O}_{5} / \mathrm{ha}$ \\
\hline & $\mathbf{P}_{2}$ & $=$ & $60 \mathrm{~kg} \mathrm{P}_{2} \mathrm{O}_{5} / \mathrm{ha}$ \\
\hline & $\mathbf{P}_{3}$ & $=$ & $90 \mathrm{~kg} \mathrm{P}_{2} \mathrm{O}_{5} / \mathrm{ha}$ \\
\hline & \multicolumn{3}{|c|}{ b. Sources of $\mathbf{P}: 02$} \\
\hline & $\mathbf{S}_{1}$ & $=$ & MAP \\
\hline & $\mathbf{S}_{2}$ & $=$ & DAP \\
\hline & \multicolumn{3}{|c|}{ c. FYM : 02} \\
\hline & $\mathbf{M}_{0}$ & $=$ & $0 \mathrm{t} / \mathrm{ha}$ \\
\hline & $\mathbf{M}_{1}$ & $=$ & $10 \mathrm{t} / \mathrm{ha}$ \\
\hline
\end{tabular}

Table.5 Effect of moisture regime and combinations of FYM and levels of P carriers on gluten content in wheat grain at harvest

\begin{tabular}{|c|c|c|c|}
\hline \multirow{2}{*}{ Treatments } & \multicolumn{3}{|c|}{ Gluten content (\%) } \\
\hline & $\mathbf{W}_{\mathbf{1}}$ & $\mathbf{W}_{\mathbf{2}}$ & $\mathbf{W}_{\mathbf{3}}$ \\
\hline $\mathbf{P}_{\mathbf{1}} \mathbf{S}_{\mathbf{1}} \mathbf{M}_{\mathbf{0}}$ & 27.18 & 33.87 & $\mathbf{2 3 . 4 9}$ \\
\hline $\mathbf{P}_{\mathbf{1}} \mathbf{S}_{\mathbf{1}} \mathbf{M}_{\mathbf{1}}$ & 28.24 & 31.57 & $\mathbf{2 9 . 1 6}$ \\
\hline $\mathbf{P}_{\mathbf{1}} \mathbf{S}_{\mathbf{2}} \mathbf{M}_{\mathbf{0}}$ & 27.73 & 21.26 & $\mathbf{2 3 . 3 5}$ \\
\hline $\mathbf{P}_{\mathbf{1}} \mathbf{S}_{\mathbf{2}} \mathbf{M}_{\mathbf{1}}$ & 30.76 & 34.89 & $\mathbf{2 7 . 0 4}$ \\
\hline $\mathbf{P}_{\mathbf{2}} \mathbf{S}_{\mathbf{1}} \mathbf{M}_{\mathbf{0}}$ & 30.32 & 26.24 & $\mathbf{2 0 . 4 7}$ \\
\hline $\mathbf{P}_{\mathbf{2}} \mathbf{S}_{\mathbf{1}} \mathbf{M}_{\mathbf{1}}$ & 30.52 & 26.53 & $\mathbf{2 5 . 1 0}$ \\
\hline $\mathbf{P}_{\mathbf{2}} \mathbf{S}_{\mathbf{2}} \mathbf{M}_{\mathbf{0}}$ & 29.46 & 29.22 & $\mathbf{2 3 . 6 1}$ \\
\hline $\mathbf{P}_{\mathbf{2}} \mathbf{S}_{\mathbf{2}} \mathbf{M}_{\mathbf{1}}$ & 35.17 & 37.54 & $\mathbf{3 3 . 0 4}$ \\
\hline $\mathbf{P}_{\mathbf{3}} \mathbf{S}_{\mathbf{1}} \mathbf{M}_{\mathbf{0}}$ & 33.43 & 29.51 & $\mathbf{2 1 . 7 2}$ \\
\hline $\mathbf{P}_{\mathbf{3}} \mathbf{S}_{\mathbf{1}} \mathbf{M}_{\mathbf{1}}$ & 34.99 & 39.42 & $\mathbf{3 2 . 0 9}$ \\
\hline $\mathbf{P}_{\mathbf{3}} \mathbf{S}_{\mathbf{2}} \mathbf{M}_{\mathbf{0}}$ & 32.21 & 24.20 & $\mathbf{2 1 . 3 9}$ \\
\hline $\mathbf{P}_{\mathbf{3}} \mathbf{S}_{\mathbf{2}} \mathbf{M}_{\mathbf{1}}$ & 31.84 & 27.28 & $\mathbf{2 4 . 2 6}$ \\
\hline & & $\mathbf{S . E m . \pm}$ & $\mathbf{C . D .} \mathbf{a t} \mathbf{5}$ \\
\hline & & 0.906 & $\mathbf{3 . 5 5}$ \\
\hline & $\mathbf{W}$ & 1.205 & $\mathbf{3 . 4 0}$ \\
\hline & & $\mathbf{2 . 0 8 7}$ & $\mathbf{5 . 8 9}$ \\
\hline
\end{tabular}


Table.6 Effect of moisture regime and combinations of FYM and levels of P carriers on length of ear head, number of grains/earhead, 1000 -grain weight, grain yield and straw yield of wheat at harvest

\begin{tabular}{|c|c|c|c|c|c|c|c|c|c|c|c|c|c|c|c|}
\hline \multirow[t]{2}{*}{ Treat. } & \multicolumn{3}{|c|}{$\begin{array}{l}\text { Length of ear head } \\
\qquad(\mathrm{cm})\end{array}$} & \multicolumn{3}{|c|}{$\begin{array}{c}\text { Number of } \\
\text { grains/earhead }\end{array}$} & \multicolumn{3}{|c|}{$\begin{array}{l}\text { 1000-grain weight } \\
\text { (g) }\end{array}$} & \multicolumn{3}{|c|}{$\begin{array}{l}\text { Grain yield } \\
\text { (kg/ha) }\end{array}$} & \multicolumn{3}{|c|}{$\begin{array}{l}\text { Straw yield } \\
\text { (kg/ha) }\end{array}$} \\
\hline & $\mathbf{W}_{1}$ & $\mathbf{W}_{2}$ & $\mathbf{W}_{3}$ & $\mathrm{~W}_{1}$ & $\mathbf{W}_{2}$ & $\mathbf{W}_{3}$ & $\mathrm{~W}_{1}$ & $\mathbf{W}_{2}$ & $\mathbf{W}_{3}$ & $\mathrm{~W}_{1}$ & $\mathbf{W}_{2}$ & $\mathbf{W}_{\mathbf{3}}$ & $\mathrm{W}_{1}$ & $\mathbf{W}_{2}$ & $\mathbf{W}_{\mathbf{3}}$ \\
\hline $\mathbf{P}_{1} \mathbf{S}_{1} \mathbf{M}_{0}$ & 7.65 & 9.53 & 6.62 & 47 & 58 & 40 & 50.50 & 61.35 & 43.70 & 3596 & 4481 & 3111 & 8219 & 10241 & 7151 \\
\hline $\mathbf{P}_{1} \mathbf{S}_{1} \mathbf{M}_{1}$ & 7.94 & 8.89 & 8.21 & 38 & 54 & 50 & 52.50 & 58.56 & 54.23 & 3735 & 4170 & 3861 & 8488 & 9528 & 8778 \\
\hline $\mathbf{P}_{1} \mathbf{S}_{2} \mathbf{M}_{0}$ & 7.82 & 5.98 & 6.57 & 48 & 36 & 40 & 51.57 & 39.50 & 43.35 & 3670 & 2812 & 3087 & 8432 & 6457 & 7087 \\
\hline $\mathbf{P}_{1} \mathbf{S}_{2} \mathbf{M}_{1}$ & 8.66 & 9.81 & 7.62 & 53 & 60 & 46 & 57.90 & 64.72 & 50.28 & 4071 & 4608 & 3580 & 9259 & 10537 & 8151 \\
\hline $\mathbf{P}_{2} \mathbf{S}_{1} \mathbf{M}_{0}$ & 8.53 & 7.37 & 5.76 & 52 & 44 & 35 & 56.37 & 48.59 & 38.12 & 4012 & 3463 & 2709 & 9170 & 7920 & 6225 \\
\hline $\mathbf{P}_{2} \mathbf{S}_{1} \mathbf{M}_{1}$ & 8.59 & 7.46 & 7.07 & 53 & 45 & 43 & 56.66 & 49.24 & 46.68 & 4040 & 3506 & 3324 & 9235 & 7969 & 7596 \\
\hline $\mathbf{P}_{2} \mathbf{S}_{2} \mathbf{M}_{0}$ & 8.28 & 8.22 & 6.65 & 50 & 50 & 40 & 54.76 & 54.23 & 43.92 & 3898 & 3861 & 3127 & 8923 & 8787 & 7127 \\
\hline $\mathbf{P}_{2} \mathrm{~S}_{2} \mathbf{M}_{1}$ & 9.90 & 10.64 & 9.29 & 60 & 64 & 57 & 65.31 & 69.52 & 61.35 & 4654 & 4957 & 4367 & 10626 & 11395 & 10024 \\
\hline $\mathbf{P}_{3} \mathbf{S}_{1} \mathbf{M}_{0}$ & 9.38 & 8.31 & 6.12 & 58 & 51 & 37 & 62.11 & 54.81 & 40.40 & 4426 & 3901 & 2876 & 10124 & 8926 & 6605 \\
\hline $\mathbf{P}_{3} \mathrm{~S}_{1} \mathbf{M}_{1}$ & 9.91 & 11.09 & 9.05 & 60 & 68 & 55 & 64.19 & 73.18 & 59.68 & 4664 & 5210 & 4250 & 10661 & 11969 & 8966 \\
\hline $\mathbf{P}_{3} \mathbf{S}_{2} \mathbf{M}_{0}$ & 9.07 & 6.80 & 6.03 & 55 & 41 & 37 & 59.88 & 44.90 & 39.78 & 4262 & 3198 & 2833 & 9780 & 7306 & 6475 \\
\hline $\mathbf{P}_{3} \mathbf{S}_{2} \mathbf{M}_{1}$ & 8.97 & 7.67 & 6.82 & 55 & 47 & 41 & 57.77 & 50.62 & 45.05 & 4216 & 3605 & 3207 & 9682 & 8234 & 6948 \\
\hline \multicolumn{2}{|l|}{-} & S.Em. \pm & CD5\% & CD5\% & S.Em. \pm & CD5\% & - & S.Em. \pm & CD5\% & - & S.Em. \pm & CD5\% & - & S.Em. \pm & CD5\% \\
\hline \multicolumn{2}{|l|}{$\mathbf{W}$} & 0.247 & 0.97 & $\mathbf{W}$ & 1.310 & 5.14 & $\mathbf{W}$ & 1.628 & 6.38 & $\mathbf{W}$ & 117.927 & 462.96 & $\mathbf{W}$ & 297.516 & 1168 \\
\hline \multicolumn{2}{|c|}{ TC } & 0.342 & 0.96 & $\mathbf{T C}$ & 2.201 & 6.21 & $\mathbf{T C}$ & 2.283 & 6.44 & $\mathbf{T C}$ & 159.798 & 451.29 & $\mathbf{T C}$ & 374.309 & 1057.11 \\
\hline \multicolumn{2}{|c|}{ W×TC } & 0.592 & 1.67 & WxTC & 3.812 & 10.77 & WxTC & 3.954 & 11.16 & $\mathbf{W} \times \mathbf{T C}$ & 276.778 & 781.67 & $\mathrm{~W} \times \mathrm{TC}$ & 648.321 & 1830.97 \\
\hline
\end{tabular}

*TC- Treatment combination 
Table.7 Effect of moisture regime and combinations of FYM and levels of $\mathrm{P}$ carriers on $\mathrm{P}$ content in grain and straw, $\mathrm{P}$ uptake in grain and straw and total uptake of $\mathrm{P}$ by wheat at harvest

\begin{tabular}{|c|c|c|c|c|c|c|c|c|c|c|c|c|c|c|c|}
\hline \multirow[t]{2}{*}{ Treat. } & \multicolumn{3}{|c|}{$\begin{array}{c}\text { P content in grain } \\
(\%)\end{array}$} & \multicolumn{3}{|c|}{$\begin{array}{c}\text { P uptake in grain } \\
(\mathrm{kg} / \mathrm{ha})\end{array}$} & \multicolumn{3}{|c|}{$\begin{array}{c}\text { P content in straw } \\
(\%)\end{array}$} & \multicolumn{3}{|c|}{$\begin{array}{c}\text { P uptake in straw } \\
(\mathrm{kg} / \mathrm{ha})\end{array}$} & \multicolumn{3}{|c|}{$\begin{array}{l}\text { Total uptake of P } \\
\text { (kg/ha) }\end{array}$} \\
\hline & $\mathbf{W}_{1}$ & $\mathbf{W}_{2}$ & $\mathbf{W}_{3}$ & $\mathbf{W}_{1}$ & $\mathbf{W}_{2}$ & $\mathbf{W}_{3}$ & $\mathbf{W}_{1}$ & $\mathbf{W}_{2}$ & $\mathbf{W}_{3}$ & $\mathbf{W}_{1}$ & $\mathbf{W}_{2}$ & $\mathbf{W}_{\mathbf{3}}$ & $\mathbf{W}_{1}$ & $\mathbf{W}_{2}$ & $\mathbf{W}_{\mathbf{3}}$ \\
\hline $\mathbf{P}_{1} \mathbf{S}_{1} \mathbf{M}_{0}$ & 0.24 & 0.25 & 0.27 & 8.49 & 11.13 & 8.49 & 0.17 & 0.18 & 0.20 & 14.14 & 18.51 & 14.22 & 22.63 & 29.64 & 22.70 \\
\hline $\mathbf{P}_{1} \mathbf{S}_{1} \mathbf{M}_{1}$ & 0.28 & 0.26 & 0.31 & 10.37 & 10.66 & 11.75 & 0.21 & 0.19 & 0.22 & 17.19 & 17.75 & 19.47 & 27.56 & 28.40 & 31.22 \\
\hline $\mathbf{P}_{1} \mathbf{S}_{2} \mathbf{M}_{0}$ & 0.27 & 0.27 & 0.27 & 9.73 & 7.40 & 8.20 & 0.20 & 0.19 & 0.19 & 16.29 & 12.38 & 13.72 & 26.02 & 19.78 & 21.92 \\
\hline $\mathbf{P}_{1} \mathbf{S}_{2} \mathbf{M}_{1}$ & 0.28 & 0.28 & 0.30 & 11.24 & 13.01 & 10.65 & 0.20 & 0.21 & 0.22 & 18.64 & 21.69 & 17.66 & 29.87 & 34.71 & 28.30 \\
\hline $\mathbf{P}_{2} \mathbf{S}_{1} \mathbf{M}_{0}$ & 0.37 & 0.36 & 0.42 & 14.99 & 12.33 & 11.35 & 0.27 & 0.26 & 0.30 & 24.96 & 20.56 & 19.00 & 39.96 & 32.89 & 30.36 \\
\hline $\mathbf{P}_{2} \mathbf{S}_{1} \mathbf{M}_{1}$ & 0.45 & 0.45 & 0.49 & 17.94 & 15.88 & 16.39 & 0.33 & 0.33 & 0.36 & 29.90 & 26.30 & 27.29 & 47.84 & 42.17 & 43.67 \\
\hline $\mathbf{P}_{2} \mathbf{S}_{2} \mathbf{M}_{0}$ & 0.40 & 0.38 & 0.47 & 15.96 & 14.86 & 14.85 & 0.29 & 0.28 & 0.35 & 26.64 & 24.65 & 24.66 & 42.60 & 39.51 & 39.51 \\
\hline $\mathbf{P}_{2} \mathbf{S}_{2} \mathbf{M}_{1}$ & 0.48 & 0.47 & 0.51 & 22.47 & 23.24 & 22.09 & 0.35 & 0.34 & 0.37 & 37.38 & 38.93 & 36.96 & 59.84 & 62.17 & 59.05 \\
\hline $\mathbf{P}_{3} \mathbf{S}_{1} \mathbf{M}_{0}$ & 0.44 & 0.47 & 0.45 & 19.48 & 18.40 & 12.93 & 0.32 & 0.34 & 0.33 & 32.46 & 30.68 & 21.63 & 51.95 & 49.09 & 34.55 \\
\hline $\mathbf{P}_{3} \mathbf{S}_{1} \mathbf{M}_{1}$ & 0.49 & 0.52 & 0.53 & 22.64 & 27.25 & 22.41 & 0.35 & 0.38 & 0.39 & 37.71 & 45.61 & 34.22 & 60.35 & 72.86 & 56.63 \\
\hline $\mathbf{P}_{3} \mathbf{S}_{2} \mathbf{M}_{0}$ & 0.44 & 0.44 & 0.49 & 18.74 & 13.86 & 13.84 & 0.32 & 0.32 & 0.36 & 31.33 & 23.09 & 23.05 & 50.07 & 36.95 & 36.90 \\
\hline $\mathbf{P}_{3} \mathbf{S}_{2} \mathbf{M}_{1}$ & 0.49 & 0.53 & 0.53 & 20.62 & 18.92 & 17.05 & 0.36 & 0.38 & 0.39 & 34.51 & 31.49 & 26.97 & 55.14 & 50.40 & 44.02 \\
\hline \multicolumn{2}{|l|}{-} & S.Em. \pm & CD5\% & CD5\% & S.Em. \pm & CD5\% & - & S.Em. \pm & CD5\% & - & S.Em.土 & CD5\% & - & S.Em. \pm & CD5\% \\
\hline \multicolumn{2}{|l|}{ W } & 0.005 & 0.021 & $\mathbf{W}$ & 0.608 & NS & W & 0.003 & 0.013 & W & 1.070 & NS & $\mathbf{W}$ & 1.669 & NS \\
\hline \multicolumn{2}{|c|}{ TC } & 0.012 & 0.035 & TC & 0.831 & 2.34 & TC & 0.009 & 0.025 & TC & 1.408 & 3.97 & TC & 2.225 & 6.28 \\
\hline \multicolumn{2}{|c|}{ W×TC } & 0.022 & NS & W $\times$ TC & 1.439 & NS & W $\times$ TC & 0.016 & NS & $\mathrm{W} \times \mathrm{TC}$ & 2.440 & NS & W $\times$ TC & 3.853 & NS \\
\hline
\end{tabular}


Table.8 Effect of moisture regime and combinations of FYM and levels of P carriers on BD, MWHC, EC (1: 2.5), pH (1: 2.5) and organic carbon of soil after harvest of wheat

\begin{tabular}{|c|c|c|c|c|c|c|c|c|c|c|c|c|c|c|c|}
\hline \multirow[t]{2}{*}{ Treat. } & \multicolumn{3}{|c|}{$\begin{array}{c}\text { BD } \\
\left(\mathrm{Mg} / \mathrm{m}^{3}\right)\end{array}$} & \multicolumn{3}{|c|}{$\begin{array}{c}\text { MWHC } \\
(\%)\end{array}$} & \multicolumn{3}{|c|}{$\begin{array}{c}\text { EC } \\
(\mathrm{dS} / \mathrm{m})\end{array}$} & \multicolumn{3}{|c|}{ pH } & \multicolumn{3}{|c|}{$\begin{array}{c}\text { Organic carbon } \\
(\%)\end{array}$} \\
\hline & $\mathbf{W}_{1}$ & $\mathbf{W}_{2}$ & $\mathbf{W}_{3}$ & $\mathbf{W}_{1}$ & $\mathbf{W}_{2}$ & $\mathbf{W}_{3}$ & $\mathbf{W}_{1}$ & $\mathbf{W}_{2}$ & $\mathbf{W}_{3}$ & $\mathbf{W}_{1}$ & $\mathbf{W}_{2}$ & $\mathbf{W}_{3}$ & $\mathbf{W}_{1}$ & $\mathbf{W}_{2}$ & $\mathbf{W}_{3}$ \\
\hline $\mathbf{P}_{1} \mathbf{S}_{1} \mathbf{M}_{0}$ & 1.66 & 1.66 & 1.67 & 21.94 & 21.76 & 21.89 & 0.17 & 0.17 & 0.16 & 7.60 & 7.53 & 7.57 & 0.35 & 0.35 & 0.34 \\
\hline $\mathbf{P}_{1} \mathrm{~S}_{1} \mathbf{M}_{1}$ & 1.63 & 1.62 & 1.63 & 22.39 & 22.34 & 22.43 & 0.19 & 0.17 & 0.17 & 7.33 & 7.23 & 7.43 & 0.36 & 0.37 & 0.37 \\
\hline $\mathbf{P}_{1} \mathbf{S}_{2} \mathbf{M}_{0}$ & 1.67 & 1.67 & 1.67 & 21.85 & 21.89 & 21.89 & 0.16 & 0.16 & 0.16 & 7.57 & 7.60 & 7.70 & 0.35 & 0.34 & 0.35 \\
\hline $\mathbf{P}_{1} \mathrm{~S}_{2} \mathbf{M}_{1}$ & 1.62 & 1.63 & 1.63 & 22.48 & 22.43 & 22.43 & 0.17 & 0.18 & 0.18 & 7.30 & 7.37 & 7.23 & 0.36 & 0.36 & 0.36 \\
\hline $\mathbf{P}_{2} \mathbf{S}_{1} \mathbf{M}_{0}$ & 1.67 & 1.67 & 1.67 & 22.09 & 21.72 & 21.62 & 0.17 & 0.16 & 0.16 & 7.50 & 7.67 & 7.57 & 0.35 & 0.35 & 0.35 \\
\hline $\mathbf{P}_{2} \mathbf{S}_{1} \mathbf{M}_{1}$ & 1.62 & 1.61 & 1.61 & 22.46 & 22.48 & 22.52 & 0.16 & 0.18 & 0.15 & 7.23 & 7.33 & 7.33 & 0.36 & 0.36 & 0.37 \\
\hline $\mathbf{P}_{2} \mathbf{S}_{2} \mathbf{M}_{0}$ & 1.66 & 1.67 & 1.66 & 22.09 & 21.76 & 21.94 & 0.17 & 0.16 & 0.16 & 7.67 & 7.60 & 7.50 & 0.34 & 0.34 & 0.35 \\
\hline $\mathrm{P}_{2} \mathrm{~S}_{2} \mathrm{M}_{1}$ & 1.62 & 1.62 & 1.63 & 22.39 & 22.52 & 22.34 & 0.17 & 0.18 & 0.17 & 7.27 & 7.27 & 7.37 & 0.36 & 0.36 & 0.36 \\
\hline $\mathbf{P}_{\mathbf{3}} \mathbf{S}_{\mathbf{1}} \mathbf{M}_{\mathbf{0}}$ & 1.67 & 1.67 & 1.67 & 21.81 & 21.89 & 21.71 & 0.16 & 0.16 & 0.17 & 7.50 & 7.57 & 7.57 & 0.34 & 0.34 & 0.35 \\
\hline $\mathbf{P}_{3} \mathbf{S}_{1} \mathbf{M}_{1}$ & 1.62 & 1.63 & 1.61 & 22.43 & 22.14 & 22.43 & 0.18 & 0.16 & 0.17 & 7.23 & 7.17 & 7.33 & 0.37 & 0.36 & 0.36 \\
\hline $\mathbf{P}_{3} \mathbf{S}_{2} \mathbf{M}_{0}$ & 1.66 & 1.66 & 1.67 & 21.72 & 21.90 & 21.67 & 0.16 & 0.17 & 0.17 & 7.60 & 7.57 & 7.60 & 0.34 & 0.34 & 0.34 \\
\hline $\mathbf{P}_{3} \mathbf{S}_{2} \mathbf{M}_{1}$ & 1.61 & 1.63 & 1.61 & 22.34 & 22.39 & 22.48 & 0.17 & 0.17 & 0.17 & 7.27 & 7.27 & 7.20 & 0.36 & 0.36 & 0.37 \\
\hline- & & S.Em. \pm & CD5\% & CD5\% & S.Em. \pm & CD5\% & - & S.Em. \pm & CD5\% & - & S.Em. \pm & CD5\% & - & S.Em. \pm & CD5\% \\
\hline $\mathbf{W}$ & & 0.003 & NS & $\mathbf{W}$ & 0.056 & NS & $\mathbf{W}$ & 0.003 & NS & $\mathbf{W}$ & 0.041 & NS & $\mathbf{W}$ & 0.001 & NS \\
\hline TC & & 0.006 & 0.018 & TC & 0.089 & 0.25 & TC & 0.008 & NS & TC & 0.053 & 0.14 & TC & 0.002 & 0.007 \\
\hline $\mathbf{W} \times \mathbf{T}$ & & 0.011 & NS & W $\times$ TC & 0.154 & NS & W $\times$ TC & 0.014 & NS & $\mathrm{W} \times \mathrm{TC}$ & 0.091 & NS & W $\times$ TC & 0.004 & NS \\
\hline
\end{tabular}


Table.9 Effect of moisture regime and combinations of FYM and levels of P carriers on available $\mathrm{N}$, available $\mathrm{P}_{2} \mathrm{O}_{5}$ and available $\mathrm{K}_{2}$ of soil after harvest of wheat

\begin{tabular}{|c|c|c|c|c|c|c|c|c|c|}
\hline \multirow[t]{2}{*}{ Treat. } & \multicolumn{3}{|c|}{$\begin{array}{l}\text { Available nitrogen } \\
\text { (kg/ha) }\end{array}$} & \multicolumn{3}{|c|}{$\begin{array}{c}\text { Available } \mathrm{P}_{2} \mathrm{O}_{5} \\
\text { (kg/ha) }\end{array}$} & \multicolumn{3}{|c|}{$\begin{array}{c}\text { Available } \mathrm{K}_{2} \mathrm{O} \\
(\mathrm{kg} / \mathrm{ha})\end{array}$} \\
\hline & $\mathbf{W}_{1}$ & $\mathbf{W}_{2}$ & $\mathbf{W}_{3}$ & $\mathbf{W}_{1}$ & $\mathbf{W}_{2}$ & $\mathbf{W}_{3}$ & $\mathbf{W}_{1}$ & $\mathbf{W}_{2}$ & $\mathbf{W}_{3}$ \\
\hline $\mathbf{P}_{1} \mathbf{S}_{1} \mathbf{M}_{0}$ & 129.22 & 134.91 & 148.56 & 29.66 & 30.97 & 34.10 & 151.28 & 157.95 & 173.93 \\
\hline $\mathbf{P}_{1} \mathbf{S}_{1} \mathbf{M}_{1}$ & 154.34 & 140.90 & 166.68 & 35.43 & 32.34 & 38.26 & 180.69 & 164.95 & 195.14 \\
\hline $\mathbf{P}_{2} \mathbf{S}_{1} \mathbf{M}_{0}$ & 137.70 & 151.47 & 155.60 & 31.61 & 34.77 & 35.72 & 161.21 & 177.33 & 182.17 \\
\hline $\mathbf{P}_{2} \mathbf{S}_{1} \mathbf{M}_{1}$ & 161.61 & 154.04 & 167.67 & 37.10 & 35.36 & 38.49 & 189.21 & 180.34 & 196.30 \\
\hline $\mathbf{P}_{2} \mathbf{S}_{2} \mathbf{M}_{0}$ & 149.45 & 159.61 & 159.74 & 34.31 & 36.64 & 36.67 & 174.96 & 186.86 & 187.02 \\
\hline $\mathbf{P}_{3} \mathbf{S}_{2} \mathbf{M}_{0}$ & 151.49 & 174.03 & 175.34 & 34.78 & 39.95 & 40.25 & 177.36 & 203.75 & 205.27 \\
\hline $\mathbf{P}_{3} \mathbf{S}_{2} \mathbf{M}_{1}$ & 171.11 & 175.99 & 175.93 & 39.28 & 40.40 & 40.39 & 200.33 & 206.04 & 205.97 \\
\hline \multicolumn{2}{|c|}{ - } & S.Em. \pm & CD5\% & CD5\% & S.Em. \pm & CD5\% & - & S.Em.. & CD5\% \\
\hline \multicolumn{2}{|c|}{ W } & 3.138 & NS & $\mathbf{W}$ & 0.724 & NS & $\mathbf{W}$ & 3.674 & NS \\
\hline \multicolumn{2}{|c|}{$\mathbf{T C}$} & 5.595 & 15.80 & $\mathbf{T C}$ & 1.284 & 3.62 & TC & 6.550 & 18.50 \\
\hline \multicolumn{2}{|c|}{ WxTC } & 9.691 & NS & W×TC & 2.224 & NS & W $\times$ TC & 11.346 & NS \\
\hline
\end{tabular}


It is obvious that treatment receiving FYM as an organic source, showed the positive effect. Singh et al., (2007) noted that such type of beneficial effect on physical fertility due to addition of organic matter due to creation of environment conductive for formation of humic acids. Stimulated the activity of soil microorganisms favours the organic carbon and reduction in bulk density. The bulk density is decreasing this could be attributed to mixing of the low density material (FYM) with the dense mineral fraction of soil (Bajpai et al., 2006).

The perusal of data with respect to available $\mathrm{N}$ and available $\mathrm{K}_{2} \mathrm{O}$ content in soil after harvest of wheat show that combination $\mathrm{P}_{1} \mathrm{~S}_{2} \mathrm{M}_{1}$ resulted in the highest available $\mathrm{N}$ $(177.30 \mathrm{~kg} / \mathrm{ha})$ and available $\mathrm{K}_{2} \mathrm{O}(207.57$ $\mathrm{kg} / \mathrm{ha}$ ) in soil after harvest of wheat, but it was statistically at par with combinations $\mathrm{P}_{3} \mathrm{~S}_{2} \mathrm{M}_{1}, \mathrm{P}_{3} \mathrm{~S}_{2} \mathrm{M}_{0}, \mathrm{P}_{3} \mathrm{~S}_{1} \mathrm{M}_{1}$ and $\mathrm{P}_{2} \mathrm{~S}_{2} \mathrm{M}_{1}$ (Table 9). Whereas, treatment combination $P_{1} S_{1} M_{1}$ proved its superiority through registering higher value of available $\mathrm{P}_{2} \mathrm{O}_{5}(40.70 \mathrm{~kg} / \mathrm{ha})$. Numerically, maximum value of available $\mathrm{N}$ (202.56 kg/ha), available $\mathrm{P}_{2} \mathrm{O}_{5}(46.50 \mathrm{~kg} / \mathrm{ha})$ and available $\mathrm{K}_{2} \mathrm{O}(237.15 \mathrm{~kg} / \mathrm{ha})$ in soil after harvest under $\mathrm{W}_{2} \mathrm{P}_{1} \mathrm{~S}_{2} \mathrm{M}_{1}$ i.e. application of $\mathrm{P}$ @ $30 \mathrm{~kg} / \mathrm{ha}$ as DAP and FYM @ $10 \mathrm{t} / \mathrm{ha}$ with 100-50 per cent available water (Table 9).

In the loamy sand (Typic Ustipsamments) of North Gujarat, maintenance of moisture regimes at 100-50 per cent available water capacity and an application of FYM @ 10 t/ha along with $60 \mathrm{~kg} \mathrm{P}_{2} \mathrm{O}_{5} /$ ha through DAP apart from recommended dose of $120 \mathrm{~kg} \mathrm{~N} /$ ha was found more advantageous in terms of yield, gluten content, $\mathrm{P}$ uptake by wheat and soil fertility status.

\section{References}

Al Harbi, S.F., Ghoneim, A.M., Modaihsh, A.S. and Mahjoub, M.O. (2013). Effect of Foliar and Soil Application of Phosphorus on Phosphorus Uptake, Use Efficiency and Wheat Grain Yield in Calcareous Soil. Journal of Applied Sciences. 13 (1): 188-192.

Bajpai, R.K., Chitale, S., Upadhyaya, S.K. and Urkurkar, J.S. (2006). Long-term studies on soil physico-chemical properties and productivity of ricewheat system as influenced by integrated nutrient management in Inceptisol of Chhattisgarh. Journal of Indian Society of Soil Science. 54 (1): 24-29.

Culley, J.L.B. (1993). Density and compressibility in soil sampling and methods of analysis (Carter, M.R. Ed.). Canadian Society of Soil Science, Lewis publishers, London. pp. 529-539.

Golakiya, B.A. (1988). Ph.D. Thesis, Gujarat Agricultural University, Junagadh Campus, Junagadh.

Jackson, M.L. (1973). Soil chemical analysis, Prentice-Hall of India Private Ltd., New Delhi.

Jackson, M.L. (1978). Soil chemical analysis, Prentice-Hall of India Private Ltd., New Delhi.

Jazy, H.D., Namini, K.N. and Ameri, M. (2012). Effect of deficit irrigation regimes on yield, yield components and some quality traits of three bread wheat cultivars (Triticum aestivum L.). International Journal of Agriculture and Crop Sciences. 4 (5): 234-237.

Olsen, S.R., Cole, G.V., Watanabe, F.S. and Dean, L.A. (1954). Estimation of available phosphorus in soil by extraction with sodium bicarbonate. U.S. Department of Agriculture. Cir. No. 939: 19.

Patel, M.S., Pavaya, R.P., Patel, V.R. and Patel, M.K. (1994). Retention and release of phosphorus in soils of Gujarat. Phosphorus in Gujarat Agriculture. 55-63. 
Piper, C.S. (1966). Soil and Plant Analysis. Hans Publishers, Bombay.

Sanyal, S.K., Dwivedi, B.S., Singh, V.K., Majumdar, K., Datta, S.C., Pattanayak, S.K. and Annapurna, K. (2015). Phosphorus in relation to dominant cropping sequences in India: chemistry, fertility relation and management option. Curent Science. 108: 12621270.

Shahi, S.H., Kushwaha, I.K. and Sharma, P.K. (2015) Effect of phosphorus levels, Phosphate Solubilizing Bacteria (PSB) and Farmyard Manure (FYM) on physico-chemical properties of soil and performance of wheat crop (Triticum aestivum L.). Indian Journal of Agriculture and Allied Sciences. 1 (1): 38-44.

Singh, G., Jalota, S.K. and Singh, Yadvinder
(2007). Manuring and residue management effects on physical properties of a soil under the rice wheat system in Punjab, India. Soil and Tillage Research. 94: 229-238.

Singh, R. and Agrawal, S.K. (2005). Effect of levels of farm yard manure and nitrogen fertilizer on grain yield and use efficiency of nutrients on wheat (Triticum aestivum). Indian Journal of Agricultural Science. 75 (7): 408-413.

Subbiah, B.V. and Asija, G.L. (1956). A rapid procedure for determination of available nitrogen in soil. Current Science. 25: 259-260.

Swaminathan, M., Kantha Josheph Rao, N., Chandramani, S.V., Subramanyam, L. and Indira, K. (1981). Balanced diet and nutritive values of common recipes. $2^{\text {nd }}$ Feb., Sharda Press, Mangalore.

\section{How to cite this article:}

Patel, D.A., K.N. Patel, R.P. Pavaya and Patel, V.R. 2019. Effect of Moisture Regimes, FYM and Levels of P Carriers on Yield, Quality and P Uptake by Wheat in Loamy Sand. Int.J.Curr.Microbiol.App.Sci. 8(08): 1088-1099. doi: https://doi.org/10.20546/ijcmas.2019.808.127 